Anna Forné *

Universidad de

Gotemburgo, Suecia

\section{Narrar la derrota: el testimonio revolucionario a partir del nuevo milenio}

\author{
Narrating the defeat: the revolutionary testimony \\ from the new millennium
}

\section{Resumen}

Unilo de lábula

Universidad Nacional del Litoral, Argentina ISSN-e: 2362-5651

Periodicidad semestral, julio-diciembre, 2021 vol. 19, núm. 22, e0008, revistaelhilodelafabula@ fhuc.unl.edu.ar

Recepción: 14072021 Aprobación: 24092021

URL: http://portal. amelica.org/ameli/ jatsRepo/247/2472702005/ index.html DOI: https://doi. org/10.14409/hf.19.22. e0008

Esta obra está bajo una Licencia Creative Commons Atribución-NoComercialCompartirlgual 4.0 Internacional.
En el cambio del milenio, las narrativas de éxito características de las primeras décadas de la Revolución cubana -las del hombre nuevo y las historias de vida heroicas - en definitiva habían perdido vigencia y poder de convicción. En cambio, dominaba el desencantamiento y una sensación general de alejamiento de las formas anteriores que ordenaban la realidad, así como una simultánea apertura y búsqueda de nuevas formas de narrar la memoria y la historia. Estas nuevas sensibilidades también se acogen a la hora de repensar, desde Casa de las Américas, las modalidades del género testimonial que la institución cultural cubana institucionalizó con la creación de un premio literario en esta categoría en 1970. En este artículo se analizan dos textos ganadores del premio testimonio de Casa de las América, Mañana es lejos (memorias verdes de los años rabiosos) (2009) de Eduardo Rosenzvaig y Su paso (2011) de Carlos E. Bishoff, con el fin de interrogar las formas de escribir testimonio a partir del 2000.

\section{Palabras clave}

testimonio, autoficción, desencantamiento, memoria, Casa de las Américas.

\section{Abstract}

At the turn of the millennium, the stories of triumph, characteristic of the first decades of the Cuban Revolution - those of the new man and the heroic life stories - had ultimately lost their validity and power of conviction. Instead, disenchantment and a general sense of detachment from the previous forms of constructing reality, as well as a simultaneous openness and a search for new ways of narrating memory and history dominated. These new sensibilities are also embraced by Casa de las Américas, when rethinking the modalities of the testimonial genre, institutionalized by the Cuban cultural institution along with the creation of a literary award in this category in 1970. In this article, two Casa de las América testimonial award winning texts are analyzed: Mañana es lejos (memorias verdes de los años rabiosos) (2009) by Eduardo Rosenzvaigand Su paso(2011) by Carlos E. Bishoff, in order to look into the ways of writing testimony after 2000.

\section{Keywords}

testimony, autofiction, disenchantment, memory, Casa de las Américas. 
I.

Alain Badiou abre El siglo con las preguntas ¿qué es un siglo? y ¿cuál es el instante de excepción que borra el siglo XX? Según Badiou, desde un punto de vista histórico-político, el siglo XX podría resumirse como un «entrecruzamiento del siglo totalitario, el siglo soviético y el siglo liberal» (2009:14). Cronológicamente aquí nos atañe el segundo, que se cierra con la caída de la URSS en 1989, momento cuando Fidel Castro se convierte en «un superviviente exótico» (2009:14) en la fila de líderes totalitarios comunistas. En la historia moderna de Cuba, la época de crisis socio-económica que la isla sufre en los años 90, como consecuencia del colapso de la unión soviética, es conocida como el «período especial en tiempos de paz» (1989-2005), que sería este instante de excepción que cancela el siglo XX. En este sentido, se podría decir que el siglo XXI arranca ya en 1990, cuando es necesario empezar a repensar desde Cuba la Revolución y, como consecuencia, reformular el imaginario revolucionario, así como las narrativas sobre la Revolución. ${ }^{1}$ En este artículo analizaré dos textos ganadores del premio testimonio de Casa de las América, Mañana es lejos (memorias verdes de los años rabiosos) (2009) de Eduardo Rosenzvaig y Su paso (2011) de Carlos E. Bishoff, con el fin de abordar cómo se reconfigura este imaginario, y con él las formas de escribir testimonio, a partir del 2000.

En el cambio del milenio, las narrativas de éxito características de las primeras décadas de la Revolución -las del hombre nuevo y las historias de vida heroicas- en definitiva habían perdido su vigencia y su poder de convicción. En La Gaceta de Cuba, publicación de la UNEAC, Ambrosio Fornet discute la reformulación del canon que se dio en este periodo y constata que el cambio socio-económico brusco del 89 tenía los mismos efectos en los imaginarios y en las expresiones simbólicas que la Revolución, solo que: «Entonces nos había tocado ganar y ahora nos tocaba perder» (Fornet, 2002:20). El autor esboza un panorama de la literatura cubana del período especial señalando un paso del ámbito de lo público y colectivo al de lo privado e individual, acompañado por un esteticismo discursivo extremado (22). Según el autor, los cambios sociales profundos a partir de la desaparición del bloque socialista en Europa obligaron a los miembros de la generación de la Revolución «sumergidos en el sueño teleológico del mejor de los mundos [...] a despertar abruptamente y encarar la pesadilla de la realidad» (20). ${ }^{2}$ En efecto, este desencantamiento, plasmado narrativamente a través de la quiebra de la épica heroica del hombre nuevo, se produce simultáneamente en otras literaturas del continente latinoamericano, una tendencia literaria que Ana María Amar Sánchez singulariza como las narrativas de la derrota, ${ }^{3}$ pobladas por héroes perdedores y antihéroes que encaran la pérdida asumiéndola como actitud ética y posición política:

\footnotetext{
El «resto», lo que queda de las guerras, revoluciones, proyectos históricos derrotados, incluye a los perdedores, a diferentes tipos de perdedores que arrastran consigo el dolor, la memoria, la necesidad de vencer el olvido o de refugiarse en él, la capacidad de resistencia o de adaptación a las condiciones impuestas por el vencedor. [...] esa situación que exige una toma de decisión del sujeto, para sostener la memoria de lo ocurrido y la propia identidad. (Amar Sánchez, 2010:27)
}

Esta suerte de desencantamiento también la examina Beatriz Cortez en relación con la sensibilidad de posguerra en Nicaragua, El Salvador y Guatemala. En Estética del cinismo Cortez plantea que el cinismo y una subjetividad precaria caracterizan las producciones culturales que responden a las estructuras del sentir de la posguerra, configuradas como una alternativa a la sensibilidad utópica de los proyectos revolucionarios: «una posición que contrasta con la estética utópica de la esperanza que ha estado ligada con los procesos revolucionarios» (Cortez, 2010:24). 
A partir del cambio del milenio, la categoría genérica «testimonio» del Premio Casa de las Américas pasa a llamarse «literatura testimonial», una transformación que además de reflejar el cambio socio-cultural antes brevemente esbozado, coincide con un nuevo auge de la literatura testimonial en Latinoamérica, simultáneo al «boom» de la memoria y el giro subjetivo del campo cultural que se produce a nivel global en esta época. En Premio Casa de las Américas memoria. 2000-2004 explican Inés Casañas y Jorge Fornet que, al cumplir 40 años el certamen, se llevaron a cabo varios cambios, entre otros: «la decisión de refundar, bajo el nombre de LITERATURA TESTIMONIAL, lo que hasta entonces se conoció como testimonio, espacio que en la práctica estaba siendo ocupado - aparte de por el testimonio propiamente dicho- por géneros más o menos afines como memorias, biografías, reportajes, etc.» (Casañas y Fornet, 2004:5-6). Revisando los ganadores a partir del 2000 se puede constatar que de los seis ganadores -José Alejandro Castaño Hoyos, La isla de Morgan (2003); Edda Fabbri, Oblivion (2007); Eduardo Rosenzvaig, Mañana es lejos (memorias verdes de los años rabiosos) (2009); Carlos E. Bishoff, Su paso (2011); Nicolás Doljanin, La sombra del tío (2013); y Liliana Villanueva, Lloverá siempre. Las vidas de María Esther Gilio (2018 [2017]) - todos menos el primero son del Río de la Plata y las tres obras premiadas entre 2007-2011 abordan las memorias de las últimas dictaduras militares; Edda Fabbri sobre el Uruguay, y Rosenzvaig y Bishoff sobre la Argentina. ${ }^{4}$ Por lo tanto, aparte de una coincidencia geográfica, las obras ganadoras convergen en cuanto al período y las circunstancias históricas abordadas, coincidentes con el período de políticas de la memoria del kirchnerismo en Argentina, que significó una resemantización de la memoria colectiva tanto en el campo político como en el socio-cultural, y la resignificación del pasado reciente en Uruguay, iniciada de forma tentativa en 2000 con la «Comisión para la paz», e intensificado a partir de la asunción al gobierno del Frente Amplio en 2005. ${ }^{5}$ Sin entrar en detalles, es posible sostener que, al mismo tiempo que las nuevas políticas de la memoria resignificaron el campo socio-político de la memoria, asimismo conllevaron un cambio con respecto a las narrativas de la memoria. Por un lado, esta es la época cuando la generación de los hijos de los militantes y desaparecidos empiezan a dar nuevas formas narrativas al pasado reciente $y,{ }^{6}$ por otro, es un momento de reformulación de los contornos existenciales, de lo que Josefina Ludmer llamó la realidad-ficción y el sujeto cotidiano íntimopúblico, acompañada por la necesidad de encontrar un nuevo vocabulario para poder nombrar las nuevas condiciones de vida post-milenio:

Supongamos que el mundo ha cambiado y que estamos en otra etapa de la nación, que es otra configuración del capitalismo y otra era en la historia de los imperios. Para poder entender este nuevo mundo (y escribirlo como testimonio, documental, memoria y ficción), necesitamos un aparato diferente del que usábamos antes. Otras palabras y nociones, porque no solamente ha cambiado el mundo sino los moldes, géneros y especies en que se lo dividía y diferenciaba. Esas formas nos ordenaban la realidad: definían identidades y fundaban políticas y guerras. (Ludmer, 2010:9)

En esta época asimismo se despliegan, como apunté antes, narraciones que dejan entrever el desencantamiento del perdedor, anterior cazador de utopías, que decide contar sus memorias, situándose entre lo personal y lo político y entre lo individual y lo colectivo. Es decir, en ocasión del nuevo milenio se empezó a gestionar un nuevo tipo de testimonio que, a partir de una renovación de la postura enunciativa, transgrede los alcances convencionales del testimonio canónico hacia una narrativa más subjetiva, autoreflexiva e introspectiva. Sin desprenderse de los alcances colectivos propios del género testimonial, este nuevo formato narrativo daba cabida a una vertiente centrada en el yo que permitía a los autores narrativizar sus memorias individuales. Con base en este nuevo formato híbrido, los testimonios, convertidos en novelas testimoniales, desestabilizaban los parámetros convencionales de la referencialidad, sin por eso inferir en o comprometer el esclarecimiento de la verdad histórica. Al respecto, Ana Casas resalta que se dibuja una nueva 
zona gris, donde se despliegan una serie de textos ambiguos en los que se atraviesa lo íntimo e introspectivo con lo público y colectivo:

$[H]$ oy, más que en otras épocas, la autoficción responde a una tendencia general del arte contemporáneo, pues, asumida la imposibilidad de un referente estable -incluido el propio autor-, los creadores siguen afanándose, en plasmar sus identidades (fragmentaria y precariamente), con más intensidad incluso que en otros períodos. Emprenden así una búsqueda que va del cauce introspectivo - en el que prima la representación de lo íntimo - a formulaciones que se vinculan a la memoria colectiva y el testimonio. [...] La intensificación del giro subjetivo en las literaturas hispánicas se expresa, en suma, a través de las experiencias personales como objeto de las obras más allá de la mera inspiración, en la medida en que estas son asumidas como materia novelable o dramatizable. (Casas, 2014:13)

En este sentido, se podría argumentar que en torno al fin del milenio domina una sensación general de alejamiento de las formas anteriores que ordenaban la realidad, y una apertura y búsqueda de nuevas formas de narrar. También se acogen estas nuevas sensibilidades a la hora de repensar desde Casa de las Américas las modalidades del género testimonial que la entidad cultural cubana institucionalizó con la creación de su premio literario en esta categoría en $1970{ }^{7}$

II.

Con Mañana es lejos. Memorias verdes de los años rabiosos (2009) de Eduardo Rosenzvaig, el género testimonial definitivamente da el paso de su pauta canónica subalterna al formato letrado, que en particular caracteriza los testimonios sobre las luchas revolucionarias y la represión militar de las dictaduras en el Cono Sur. ${ }^{8}$ Ya desde el título este texto exhibe su especificidad estética; al representar los sinsentidos de lo real, que caracterizan los últimos cincuenta años de la historia argentina, recurre a la parodia y al humor, que a veces roza lo absurdo. La verborragia del relato de Rosenzvaig se refleja nítidamente en la adjetivación exagerada del título, una característica que reverbera a lo largo de la narración de las memorias del autor-narrador, y que la aparta de la austeridad protocolar del testimonio letrado-militante. En Mañana es lejos, Rosenzvaig retoma y repite una serie de temas propios del género testimonial, pero toma distancia crítica al dar un giro paródico, y a veces incluso humorístico a la denuncia. Señala Ana Casas los efectos del distanciamiento, propios del humor, en relación con el discurso autoficcional:

El humor es, en efecto, una de esas estrategias autoconscientes que rompen la transparencia del relato y dinamitan el discurso referencial que, inicialmente y en apariencia, la autoficción propone. Implica un modo de interrogarse sobre la identidad, pero quitándole solemnidad o dogmatismo al proceso introspectivo, al tiempo que expresa un irrenunciable compromiso con el propio arte. (Casas, 2015:175)

En relación con los despliegues autoficcionales en clave paródica del género testimonial, en Mañana es lejos se desfiguran la transparencia y la inmediatez propias del discurso referencialrealista y, en cambio, se articula un relato intrincado que subvierte precisamente la severidad y rigidez dogmáticas que suelen caracterizar al género testimonial. ${ }^{9}$ Paralelamente a este desmontaje también se desintegra la figura de autor testimonial, socavándose su tradicional posición privilegiada. Se trata, pues, de la parodia como una imitación crítica con fines renovadores (Casas Janices, 2015:178), en este caso de los canónicos testimonios de militancia. En el sistema literario argentino, Rosenzvaig sin duda escribe desde la periferia, y una parte del relato podría leerse 
como una declaración de amor a San Miguel de Tucumán, nombrada por Rosenzvaig como la ciudad del deseo: «Una ciudad delicuescente, licuada después de absorber tanta humedad del aire en los veranos infinitos, y que recuperaba solidez, cristalizándose, llegados los inviernos de la zafra» (Rosenzvaig, 2009:242). Por otra parte, en la historia contemporánea argentina fue en la provincia de Tucumán donde se inició oficialmente la aniquilación de los «elementos subversivos», cuando en 1975 las Fuerzas Armadas instalan el Operativo Independencia. Este es un hecho clave al cual retorna Rosenzvaig en varias ocasiones a lo largo de su relato, reinstalando momentáneamente, si bien con algo de indecisión, el tono austero de la denuncia, propio del testimonio protocolar: «...Tucumán tenía claramente dos gobiernos, uno a luz diurna con un simpático 'turco' y tránsfuga de gobernador, y la noche 'libre' para el Operativo de 'exterminio' de civiles llamado, curiosamente de la Independencia, avalado por el propio gobernador con simpatía.» (118). En este sentido, el testimonio de Rosenzvaig se escribe desde el margen, abordando un acontecimiento decisivo de la historia nacional contemporánea. No obstante, en calidad de testimoniante, Rosenzvaig no expresa afán de historiador, porque lo que se ofrece a lo largo de Mañana es lejos son fragmentos de historias de vida que tienen como protagonista principal la derrota y el derrumbe de la Argentina, un desarrollo distanciado del que había imaginado en su tiempo la generación de idealistas de la que confiesa formar parte el autor. En Mañana es lejos, los capítulos se despliegan como una galería sobreabundante de retratos de personajes y lugares claves en la historia de vida del autor-narrador, íntimamente conectada a la historia argentina.

Mañana es lejos abre con cuatro prólogos de carácter poético en los que el autor-narrador se sitúa y explicita sus intenciones, así como las carencias del relato, que se deben al olvido. «No tengo mucho para decir en este prólogo, recordarte que mis años en la dictadura, nuestros años, los años de una generación en todo caso, van con sus derrumbes» (7), aclara el autor-narrador en el segundo prólogo, para en el tercero reivindicar el poder de la palabra:

Este es un texto autobiográfico y lo que hay de otros viene a cuento de los resoplidos y silencios del autor. Ciertamente incapaz, destinado a contagiar a quienes no fuimos superhombres, el libro asegura que es posible inhalar la atmósfera delicada de la revolución hecha de una rama poética. (9)

En todos los sentidos, el texto de Rosenzvaig es generacional, y temática e ideológicamente se inscribe en la genealogía de testimonios letrados de militantes de izquierda producidos en el Cono Sur, que por lo general se dirigen a una comunidad de «escucha interna», que tiende a convertir la narrativa testimonial en una repetición ritualizada (Jelin, 2002: 86). No obstante, en cuanto a las elecciones estéticas y formales, Mañana es lejos se aparta con creces de la textura protocolar del género testimonial en tanto épica heroica, a favor de un acercamiento que podría pensarse en términos de una parodia con tintes de absurdo. Por un lado, en la primera parte de Mañana es lejos se formula, desde la bicicletería donde se refugia el narrador durante los años de la dictadura, una reflexión filosófica sobre la existencia, plasmada en forma de una suerte de flujo de conciencia, en el que se entrecruzan temporalidades y personalidades. Por el otro, una vez franqueadas estas páginas iniciales que, debido a su carácter absurdo e incoherente, obvian todas las fórmulas testimoniales canónicas, los lectores se encuentran con una muestra extensa de retratos difusos de diferentes personajes, entre los que figura el mismo yo del narrador-autor. En este sentido, el discurso testimonial referencial se distorsiona mediante la incorporación de componentes y mecanismos ajenos al género, como pueden ser un lenguaje literario rebuscado y la representación de situaciones absurdo-realistas (que de todos modos pueden poseer algo de veracidad), en fin, lo que hace a la parodia.

En las Actas del premio testimonio de Casa de las Américas de 2009 el jurado, conformado por Paco Ignacio Taibo II, Hernán Uribe Ortega y José Ignacio López Vigil, destaca los siguientes méritos de Mañana es lejos: 
[L]a inmensa capacidad para sintetizar la tragedia de la generación rebelde argentina a lo largo de veinte años, la calidad literaria del lenguaje, el brillo de las imágenes y metáforas, y su notable habilidad para narrar que la masacre no solo afectó a muertos y desaparecidos, sino que destruyó el tejido social de todo un país, en un sorprendente ejercicio de reflexión y memoria sobre un tema crucial en la América Latina... (Actas del Premio Casa de las Américas, 2009)

La colectividad testimonial de Mañana es lejos es fragmentaria y su forma fraccionada en cierto sentido refleja esta destrucción del tejido social mencionada en las actas, causada primero por la dictadura y posteriormente por las políticas neoliberales, denunciadas en el testimonio de Rosenzvaig. Esta desintegración, sufrida tanto por el colectivo como por sus integrantes individuales, es la que se representa en Mañana es lejos, por una parte por medio de la forma segmentada e incompleta de las escenas representadas y, por la otra, mediante un registro absurdo que permea todo el relato, quebrando de este modo el horizonte de expectativas del lector testimonial, acostumbrado a un discurso factual e inmediato. En Mañana es lejos, el absurdo es la forma que toma el intento fracasado de registrar lo real, o sea, la forma que elige el autor-narrador para exponer lo que no se puede entender ni simbolizar, pero que forma parte de su vida cotidiana: el horror, la desaparición, la muerte y la pobreza. Es también en relación con lo real que se autoconfigura el yo narrador-autor-personaje desde diferentes temporalidades y espacios: «...la autoficción, transida de humor, se aparta de lo real para volver a lo real, y poner de manifiesto el artificio que rige toda representación, incluida la del yo...» (Casas Janices, 2015: 182).

En el episodio inicial -«Rosenkranz el inflacionado»- el alter-ego del autor-narrador se llama Rosenkranz. En una narración confusa, la vida del protagonista se envuelve y se confunde con la de Hegel, cuando la representación de los quehaceres diarios en la bicicletería donde trabaja Rosenkranz y la narración de la cotidianeidad del barrio, se interrumpen por largas digresiones sobre la biografía de Hegel. Así, al testimoniar sus vivencias durante la dictadura, el autor se distancia de sí mismo, convirtiéndose por medio de la escritura en persona(je) de su propia autofiguración, desdoblándose en serie (Rosenzvaig/Rosenkranz/Hegel): «La llamo Rosenkranz a mi persona, no tanto para mirarme con condescendencia, como para señalar que ese nombre viaja unido a la primera biografía de un biografiado» (2009: 12). En el texto multifacético de Rosenzvaig, por partes poco accesible, sin duda se desestabilizan las bases mismas del discurso testimonial, y la voz utópica y esperanzada del hombre nuevo desaparece a favor de una voz desesperada, que por medio de la parodia y el humor desacraliza el legado militante al desenmascarar el absurdo de la historia y confesar la derrota.

La derrota y las ilusiones quebradas también son el tema del cuarto ganador del premio testimonio desde que se nombrara novela testimonial. En Su paso Carlos Bischoff relata sus memorias de las cárceles de la dictadura argentina. Montada como un diálogo entre dos personajes que comparten esta experiencia, el autornarrador (supuestamente Carlos) y su interlocutor llamado Pablo, la novela-testimonio se titula Su paso, aludiendo a las caminatas por Barcelona de estos dos sujetos, durante las cuales conversan sobre la experiencia carcelaria, principalmente en el penal Rawson. Explica Aylín Pérez Hernández que Pablo es el «otro yo» del autor, quien recurre a la tercera persona con el fin de distanciarse de la experiencia narrada: «Pablo y Carlos Bischoff son la misma persona física, aunque conviven en un solo cuerpo dos identidades, por momentos, totalmente opuestas» (Pérez Hernández, 2020: 189). En efecto, Pablo fue el nombre de guerra del autor cuando militaba en el sindicato de prensa de Córdoba, según cuenta en una entrevista con Pérez Hernández (203). Este juego de espejos se despliega en varios niveles, tiempos y espacios narrativos, combinados a fines de desarrollar una autocrítica de la militancia de los años 60 y 70 , así como una metareflexión en torno a la escritura de las memorias de la represión.

En el prólogo testimonial de rigor, el autor-narrador narra el devenir de la escritura de su testimonio después de tantos años, sancionando desde el inicio la veracidad de su historia, sostenida a pesar del tiempo transcurrido desde los acontecimientos narrados: 
De cualquier modo, la canción de Pablo - pido disculpas a Daniel Viglietti por usar el título de un tema suyoes absolutamente cierta aunque me haya tomado la libertad de incorporarle algunos hechos -muy pocos y siempre en esos casos narrados en tercera persona - de los que no fue protagonista directo. (Bischoff, 2011: 12)

Aparte de mirarse en el otro, que es el mismo, tal como la confusión de los deícticos revela, el testimonio de Carlos Bischoff invoca la idea del colectivo, propio del testimonio canónico, sugiriendo que la historia de Pablo «es la historia parecida de muchos.» (2011: 12). La novedad de este texto con respecto a los testimonios carcelarios tempranos escritos en el Cono Sur es la autocrítica desarrollada en Su paso, que alude a la poca preparación ideológica de los militantes y las consecuencias atroces de los errores cometidos. Incluso llega a sugerir, casi al pasar, que no volvería a hacer lo mismo, dado el alto precio que su generación tuvo que pagar: «... a los errores no basta con corregirlos, sino que se pagan. Y cuando se pagan como se pagaron... bueno, mejor no cometerlos» (2011: 149).

En repetidas ocasiones el autor-narrador comenta el desafío que supone narrar las memorias de la militancia y la cárcel, y en relación con el reto narrativo de narrar el terror, el miedo, así como la monotonía de la vida carcelaria propone una «receta» del testimonio de la represión política en el Cono Sur: «tal vez muchos años podrían solo concentrarse en cuatro o cinco anécdotas y a otra cosa» (2011: 89). En efecto, de la misma manera que en Oblivion de Edda Fabbri, en el relato de Carlos Bischoff, la descripción de la vida carcelaria se sostiene en la acumulación de relatos breves, anclados en lo tocable, en el objeto, o en la acción sustantivada, invocados con el fin de condensar estos largos años uniformes en unas cuantas anécdotas. Incluso acontecimientos como la acción de la requisa de las celdas se convierten en hechos casi cotidianos, y por tanto también se sustantivizan, convirtiéndolos en objetos de descripción. Gran parte de Su paso repasa de forma tediosa la vida carcelaria, compartimentada en anécdotas como, por ejemplo, «las cartas», «requisa de pabellón», «la visita», «los libros, la lectura». En este sentido, las caminatas de Pablo y su interlocutor en Su paso, que dan como resultado la escritura del testimonio, contrastan con la inmovilidad y monotonía de las anécdotas de cárcel. Así, tanto el andar, el diálogo como la escritura resultan ser formas para intentar superar el trauma de los años de represión, que al fin y al cabo el autor-narrador juzga inenarrables: «Aun mirado desde la distancia de los años, recordar experiencias que no se logran transmitir en su intensidad contando o escribiéndolas, es de terror». (2011: 147)

En La experiencia opaca (2009: 15-17) Florencia Garramuño traza una línea en la literatura argentina que en los años 70 y 80 trabaja con los restos de lo real hacia un cambio del estatuto de lo literario a través de una escritura radical, continuada en la literatura contemporánea. Garramuño toma prestado de Hélio Oiticica el neologismo egoexistenciateca de lo real para nombrar este nuevo repertorio artístico que destaca por su reelaboración de la relación entre arte y experiencia, articulada desde una subjetividad marcada, pero sin pretensiones biográficas, que desdibuja los límites genéricos y las convenciones artísticas:

Se trata de un tipo de escritura que, a pesar de hacer evidentes los restos de lo real que forman el material de sus exploraciones, se desprende violentamente de la pretensión de pintar una 〈realidad〉completa regida por un principio de totalidad estructurante. (Garramuño, 2009: 19)

Esta suerte de relatos, en los que la realidad desborda y supera al sujeto, se pueblan de pos-yoes, en el sentido de que los personajes figuran con nombres diferentes y los protagonistas se entremezclan los unos con los otros a fines de configurar una experiencia diferente (36-37). Garramuño concluye que: 
En su apertura y su vulnerabilidad ante el mundo, este tipo de arte y de escritura conjuga lógicas diferentes y desestabilizadoras que exponen una vulnerabilidad del sujeto y de la experiencia que nunca podría corresponderse con la noción de un sujeto u obra autónomos. (46)

En el caso de Mañana es lejos, se renuncia al sujeto autónomo, dueño de su experiencia y con capacidad de plasmarla en la obra literaria sin fisuras, junto al abandono de la representación vertical propia del testimonio canónico. Así, el mismo sujeto autoral y sus varios pos-yoes hablan de forma horizontal desde una zona liminal, a la que han sido desplazados como consecuencia de las políticas neoliberales. En la misma dirección, a través de la elaboración literaria y los matices psicológicos esbozados en Su paso se abandonan las certezas del género testimonial temprano, si bien de forma menos audaz y experimental.

\section{Referencias}

Actas del Premio Casa de las Américas. (2009). Casa de las Américas; Archivo de la Casa de las Américas.

Actas del Premio Casa de las Américas. (2011). Casa de las Américas; Archivo de la Casa de las Américas.

Amar Sánchez, Ana María (2010). Instrucciones para la derrota: Narrativas éticas y políticas de perdedores. Anthropos.

Badiou, Alain. (2009). El siglo. Manantial.

Basile, Teresa (2019). Infancias: La narrativa argentina de HIJOS. Eduvim.

Birkenmaier, Anke y Whitfield, Katheryn. (2011). Havana beyond the ruins: Cultural mappings after 1989. Duke University Press.

Bischoff, Carlos (2011). Su paso. Casa de las Américas.

Blejmar, Jordana (2016). Playful memories: The autofictional turn in postdictatorship Argentina. Palgrave Macmillan.

Blejmar, Jordana; Mandolessi, Silvia y Perez, Mariana Eva (2018). El pasado inasequible: Desaparecidos, hijos y combatientes en el arte y la literatura del nuevo milenio. EUDEBA.

Casañas, Inés y Fornet, Jorge (2004). Premio Casa de las Américas memoria. 2000-2004. Casa de las Américas.

Casas Janices, Ana (2014). La autoficción en los estudios hispánicos: perspectivas actuales. En El yo fabulado: Nuevas aproximaciones críticas a la autoficción (pp. 7-27). Iberoamericana; Vervuert.

Casas Janices, Ana (2015). Desmontando al autor. Ironía, parodia y sátira en la narrativa y el cine autoficcionales. Tropelías: Revista de Teoría de la Literatura y Literatura Comparada, 24, 174-190.

Castaño Hoyos, José Alejandro (2003). La isla de Morgan. Casa de las Américas.

Cortez, Beatriz (2010). Estética del cinismo: Pasión y el desencanto en la literatura centroamericana de posguerra. F\&G Editores.

Doljanin, Nicolás (2013). La sombra del Tío. Fondo Editorial Casa de las Américas.

Duchesne Winter, Juan (2010). La guerrilla narrada: Acción, acontecimiento, sujeto. Ediciones Callejón.

Fabbri, Edda (2007). Oblivion. Ediciones del Caballo Perdido.

Fernández, Damián (2000). Cuba and the Politics of Passion. University of Texas Press. 
Ferrari, Guillermina (2014). Community and Culture in Post-Soviet Cuba. Routledge.

Forcinito, Ana (2019). Intermittences: Memory, Justice, and the Poetics of the Visible in Uruguay. University of Pittsburgh Press. https://public.ebookcentral.proquest.com/choice/publicfullrecord.aspx?p=5630420

Forné, Anna (2009). La autoficción testimonial: Oblivion de Edda Fabbri. Telar. Revista del instituto interdisciplinario de estudios latinoamericanos, VI(7-8), 63-75.

Forné, Anna (2014a). El género testimonial revisitado. El premio testimonio de Casa de las Américas (1970-2007). El taco en la brea, 1(1), 216-232.

Forné, Anna (2014b). Palabras en suspenso: Notas sobre las narrativas carcelarias de Mauricio Rosencof. En Forné,

Anna \& Karlsson, Britt-Marie (Eds.), Strategies autofictionnelles / Estrategias autoficcionales (pp. 81-91). Peter Lang.

Forné, Anna (2018). The Politics of Poetics: Latin American Testimonial Literature and the Casa de las Américas Literary Prize (1970-1975). Studies in Testimony, 1(1), 31-51.

Forné, Anna (2021). Reflections on the Testimonial Genre and Casa de las Américas' Literary Award (1970-2017). Karib - Nordic Journal for Caribbean Studies, 6(1), 8. https://doi.org/10.16993/karib.69

Forné, Anna (En prensa). La honestidad con lo real: apuntes sobre las nuevas formas del testimonio a partir del 2000. En Pizarro Cortés, Carolina (Ed.), Nuevas formas del testimonio. Universidad de Santiago de Chile.

Fornet, Anna (2002). La crítica bicéfala: Un nuevo desafío. La Gaceta de Cuba, 1 (enero-febrero), 20-25.

Garramuño, Florencia (2009). La experiencia opaca: literatura y desencanto. Fondo de Cultura Económica de Argentina.

Hernandez-Reguant, Ariana (2009). Writing the Special Period: An Introduction. En Cuba in the special period: Culture and ideology in the 1990s (pp. 1-18). Palgrave Macmillan. http://www.loc.gov/catdir/enhancements/ fy0902/2008021629-b.html

Jelin, Elizabeth (2002). Los trabajos de la memoria. Siglo XXI de España Editores.

Loss, Jacqueline (2013). Dreaming in Russian: The Cuban Soviet imaginary. University of Texas Press.

Ludmer, Josefina (2010). Aquí América Latina: Una especulación. Eterna Cadencia.

Pérez Hernández, Aylen. (2020). Reconstrucción del yo, memoria colectiva y violencia en Su paso, de Carlos Bischoff. Telar. Revista de instituto interdisciplinario de estudios latinoamericanos, 24, 179-212.

Quiroga, José (2005). Cuban palimpsests. University of Minnesota Press. http://www.loc.gov/catdir/toc/ecip0515/2005019767.html

Rosenzvaig, Eduardo (2009). Mañana es lejos: Memorias verdes de los años rabiosos. Casa de las Américas.

Villanueva, Liliana (2018). Lloverá siempre. Las vidas de María Esther Gilio. Criatura Editora. 


\section{Notas}

1. En el momento en que estoy finalizando este artículo (julio de 2021), en Cuba las protestas ciudadanas en la calle aumentan.

2. Sobre la literatura y cultura cubanas a partir de los años 90, véase por ejemplo Birkenmaier \& Whitfield (2011); Fernández (2000); Ferrari (2014); HernandezReguant (2009); Loss (2013); Quiroga (2005).

3. Señala Juan Duchesne Winter con respecto a la actual desconsideración de las narraciones guerrilleras así como de la idea del foco guerrillero que: «Si en la primera década del siglo XXI la experiencia guerrillera latinoamericana solo se discute en muy contadas ocasiones, cual oscuro y vergonzante culto sigloveintista, bajo los motivos del fracaso y degradación, ¿ qué no será la concepción del foco, repudiado como semilla esencialmente ruinosa de la guerrilla por muchos de sus más notables practicantes de antaño...» (2010:12).

4. Sobre Oblivion véase Forné (2009) y sobre La isla de Morgan, Forné (En prensa).

5. Sobre el caso uruguayo véase Forcinito (2019).

6. Véase, por ejemplo, Basile, (2019); Blejmar et al., (2018); Blejmar, (2016).

7. Sobre el la fundación del premio testimonio, véase Forné (2014a, 2018, 2021).

8. Rossana Nofal establece la distinción entre testimonio canónico y testimonio letrado.

9. Otro ejemplo importante de este desliz es la obra del ex-dirigente tupamaro Mauricio Rosencof. Véase Forné (2014b). 